\title{
ON THE SIZE OF THE STRAINED REGION PRIOR TO AN EXTREME EARTHQUAKE*
}

\author{
By K. E. Buluen
}

\begin{abstract}
A previous formula of the author's has been adapted to give the volume $V$ of the region in which the material is near breaking-point, just prior to an extreme earthquake, in terms of the released energy and breaking-strength near the focus. In the light of recent earthquake energy-magnitude data of Gutenberg, Richter, and Benioff, it is inferred that $V$ is at least of the order of the volume of a sphere of radius $25 \mathrm{~km}$. Comparison with other estimates of the size of strained regions in earthquakes suggests that $V$ may possibly reach the order of the volume of a sphere of radius $50 \mathrm{~km}$. It is suggested that a factor in the occurrence of extreme earthquakes is the existence, in the zone of strain, of strength rather greater than in the majority of major earthquakes of magnitude one unit or more less than the extreme value.
\end{abstract}

1. Let $E$ be the energy released in seismic waves in an earthquake, and let $q E$ be the distortional strain energy in the focal region just prior to the occurrence of the earthquake. Let $V$ denote the volume of the strained region, assuming the material to have been at breaking-point throughout the whole volume. Then if $S$ and $\mu$ are the Mises strength and the rigidity assumed constant throughout $V$, it follows, from work in a previous paper, ${ }^{1}$ that

$$
12 q \mu E=S^{2} V
$$

On plausible assumptions, the formula (1) sets an upper bound to the value of $E / S^{2}$ in the greatest earthquakes. In paper 1 , this result was considered in relation to the tentative earthquake energy-magnitude formula of Gutenberg and Richter, ${ }^{2}$ namely,

$$
\log _{10} E=12+1.8 M
$$

which gives an energy of about $10^{27}$ ergs for the strongest earthquakes. The broad conclusion was drawn that either the energy $E$ as given by (2) is appreciably too high in large earthquakes, or that the fracture-resisting strength of the material in the focal region of the greatest deep-focus earthquakes is appreciably greater than some writers have hitherto assumed.

Professors Gutenberg, Richter, and Benioff have since kindly informed me of recent work in course of publication, according to which the formula

$$
\log _{10} E=11+1.6 M
$$

\footnotetext{
* Manuscript received for publication April 8, 1954.

${ }^{\prime}$ K. E. Bullen, "On Strain Energy and Strength in the Earth's Upper Mantle," Trans. Am. Geophys. Union, 34:107-109 (1953). This paper will be referred to as paper 1. (In paper 1, the value of the strength at breaking-point was taken as $\left.\sqrt{\left(\Sigma \Sigma P^{2} i j\right.}\right)$, where $P_{i j}$ denotes the deviatoric stress. This was sufficiently accurate for the broad deductions made in paper 1 ; but for the purposes of the present paper it is desirable to use the Mises function, equivalent to $\sqrt{\left(\Sigma \Sigma 3 P^{2} i j\right)}$, and hence the numerical factor on the left side of (1) is 12 instead of 4 .)

2 B. Gutenberg and C. F. Richter, Seismicity of the Earth and Associated Phenomena (Princeton University Press, 1951), p. 10.
} 
fits the case of large earthquakes rather better than (2), although they point out that (3) must still be regarded as tentative. In addition, Professor A. L. Hales has independently informed me (in a private communication) of his opinion, based on investigations by himself and collaborators on Witwatersrand tremors, that formula

(2) gives energies too great, for the range of magnitudes involved in these tremors, by a factor of 60 . Other opinions have been expressed, all pointing in the same direction. Thus the first of the two alternative conclusions reached in paper 1 would appear to be fairly well substantiated.

2. With the improvement from (2) to (3), the volume $V$ is probably now the most uncertain of all the quantities in (1), and it becomes worth while to discuss the order of magnitude of $V$ in more detail than was possible in paper 1.

For an earthquake of the greatest known magnitude, i.e., 8.6, (3) gives $E \approx 6 \times$ $10^{24} \mathrm{ergs}$, while the values of $S$ indicated by laboratory experiments on rocks of the type that predominate in the crustal layers are of the order of $10^{9} \mathrm{dyn} / \mathrm{cm} .{ }^{2}$ Substituting these values into (1), and taking $\mu=0.4 \times 10^{12} \mathrm{dyn} / \mathrm{cm} .^{2}$ and $q=2$, as in paper 1 , we then derive

$$
V=6 \times 10^{19} \mathrm{~cm}^{3},
$$

which is equivalent to the volume of a sphere of radius $25 \mathrm{~km}$.

The result (4) involves a reduction from an earlier rough figure ${ }^{3}$ of $10^{21} \mathrm{~cm}^{3}{ }^{3}$, a good part of the reduction being attributable to the change from (2) to (3).

3. The new result may be compared with a previous estimate ${ }^{4}$ based on a different approach, of the maximum size of the strained region just before an earthquake. (The immediate context in which the estimate was presented was concerned with deep-focus earthquakes, but that does not affect the following inferences.) The estimate was based on seismic data bearing on the maximum speed $v$ of development of fracture in the focal region and time interval $t$ during which energy is significantly transformed into seismic waves. Upper limits of $10 \mathrm{~km} / \mathrm{sec}$. (the velocity of $P$ waves at a depth near $600 \mathrm{~km}$.) and 10 sec. were assigned to $v$ and $t$, and led to a roughly estimated upper limit of the strained volume as that of a sphere of radius $100 \mathrm{~km}$., i.e., $4 \times 10^{21} \mathrm{~cm} .^{3}$ This limit is, however, probably capable of considerable reduction on two grounds. First, the distribution of strain would probably deviate markedly from spherical symmetry, so that the calculation could be expected to indicate only the greatest of the three dimensions of the strained region, the other two dimensions being probably much less. Secondly, the numerical data for $t$ (and possibly $v$ ) are probably very much on the high side except perhaps in abnormal cases (for example, the 1906 San Francisco earthquake) in which the departure from symmetry is extremely marked (but in such cases the first argument would apply with greater force). On these grounds, the upper limit to the strained region is then possibly reducible to the order of $10^{20} \mathrm{~cm} .^{3}$, though of course this estimate remains very rough.

In interpreting (4), it needs to be appreciated that $V$ has been defined on the artificially simple assumption that the whole of the strained region is at breaking-point

\footnotetext{
${ }^{3}$ K. E. Bullen, An Introduction to the Theory of Seismology, 2nd ed. (Cambridge University Press, $1953)$, p. 245.

4 "Colloquium on Plastic Flow and Deformation within the Earth," Trans. Am. Geophys. Union, $32: 526$ (1951).
} 
just before the earthquake. Thus the agreement between (4) and the final estimate of the last paragraph is as good as can be expected.

4. It is next of interest to compare the result (4) further with an estimate by Benioff (in course of publication and kindly made available by Professor Benioff to the author) of the volume of the zone of appreciable strain for the Kern County earthquake of July 21, 1952. Professor Benioff estimates the surface area of the zone to be $10^{13} \mathrm{~cm} .{ }^{2}$, and, assuming the zone to extend vertically downward from the outside surface to the Mohorovičic discontinuity at a depth of $35 \mathrm{~km}$., gives, as a rough approximation to its volume, $7 \times 10^{19} \mathrm{~cm} .^{3}$

This figure is remarkably close to the result (4). But there are two factors that have to be taken into account in the comparison: (i) it is to be expected that the material would have been rather short of breaking-point before the Kern County earthquake in a large fraction of the volume found by Benioff; (ii) the magnitude of this earthquake was one full unit short of the extreme value of 8.6. The effect of (i) would be that $V$ as defined in section 2 above would be perhaps only about onequarter or so of Benioff's volume. The effect of (ii) would be in the opposite direction, in that increasing $M$ by unity would by (3) entail increasing $E$ by a factor of 40 . Since $V$ is proportional to $E$ in (1), the net effect of (i) and (ii) would be a discrepancy of the order of a factor of 10 between Benioff's result and (4).

In all the circumstances, the discrepancy must still be regarded as quite small. Even so, there is a fairly ready way of reducing it. This is to assume in the zone of strain of the Kern County earthquake a lower value of the strength $S$ than $10^{9}$ $\mathrm{dyn} / \mathrm{cm} .^{2}$ In relation to the formula (1), a reduction by a factor of one-third would be formally sufficient. Another section of Benioff's investigation independently indicates that the average strength in the zone of strain was less than $10^{9} \mathrm{dyn} / \mathrm{cm} .{ }^{2}$ (Benioff in fact would put the strength as appreciably less than one-third of this.)

5. Further comparison may be made with an estimate of Jeffreys ${ }^{5}$ of the size of an earthquake focus for the case in which the energy is released by slipping along a plane fault face. Taking the linear dimensions of the area in which slipping occurs at a single stage to be $l \mathrm{~km}$., Jeffreys estimates that, for an ordinary fault in which pseudotachylyte is not formed, the released energy would be roughly equal to $K l^{2}$ ergs, where $K=4 \times 10^{19}$, so that for an earthquake in which $E=10^{22} \mathrm{ergs}, l$ would be $16 \mathrm{~km}$.

Direct comparison of (4) with the method of Jeffreys is probably not permissible since in extreme earthquakes there would presumably be quantities of pseudotachylyte formed, in which case the requisite value of $K$ would be appreciably larger. (The value of $K$ is proportional to $\theta^{2}$, where $\theta$ is the rise in temperature at the fault during the slip; the value $K=4 \times 10^{19}$ assumes $\theta=1,000^{\circ}$.) It is therefore necessary, in attempting a comparison, to keep to the value $E=10^{22}$ ergs used by Jeffreys. Using this value and other data as in section 2 would give $V$ equal to the volume of a cube of side-length $5 \mathrm{~km}$.

The discrepancy between the two indicated linear dimensions of $16 \mathrm{~km}$. and $5 \mathrm{~km}$. can be partly accounted for by assuming that the dimension of the originally strained zone at right angles to the fault face would be appreciably less than $16 \mathrm{~km}$. But there is the suggestion of a residual discrepancy, which, in keeping with

\footnotetext{
${ }^{5}$ H. Jeffreys, The Earth, 3rd ed. (Cambridge University Press, 1952), p. 341.
} 
Benioff's result, suggests that for most earthquakes which are one magnitude or more short of the extreme value a less value of $S$ than $10^{9} \mathrm{dyn} / \mathrm{cm} .{ }^{2}$ is relevant.

6. The discrepancies between the results of using (1) and the results in sections 3 , 4 , and 5 above are not great considering the tentative character of parts of the data used. The fact that several very distinct approaches to estimating the size of the strained region should agree within a factor of order about 10 is in itself significant, and gives good support to (4) as providing a lower limit to the volume of the heavily strained zone in an extreme earthquake.

A question remains, namely, whether on account of the indications of the work of Jeffreys and Benioff the estimate in (4) should be raised by using in (1) a value of $S$ less than $10^{9} \mathrm{dyn} / \mathrm{cm}^{2}$ The investigations of Jeffreys ${ }^{6}$ on the stress differences needed to support the earth's mountain systems imply that the strength exceeds $10^{9} \mathrm{dyn} / \mathrm{cm} .^{2}$ at least in certain parts of the crust. In the majority of earthquakes of magnitude say $7 \frac{1}{2}$ or less, and in particular the Kern County shock, it is likely that earlier seismic activity or other cause in the affected region has produced weaknesses which make the mean strength appreciably less than this figure. In the case of extreme earthquakes, on the other hand, the great strain energy accumulated may well be a consequence of the fact that the strength in the focal region is greater than in many other seismically active regions; a strength less than $10^{9} \mathrm{dyn} / \mathrm{cm} .{ }^{2}$ may well force the occurrence of earthquakes with magnitudes appreciably less than the extreme figure of 8.6 . For example, it may be significant that the two great Assam earthquakes of 1897 and 1950 occurred in the vicinity of the Himalayas where Jeffreys computes the strength to be $1.6 \times 10^{9} \mathrm{dyn} / \mathrm{cm} .{ }^{2}$ In these circumstances it would appear best not to raise the minimum estimate of the volume of the strained region provided by (4).

Some doubt remains concerning the best value to take for $q$, but the uncertainty in $q$ is not likely to affect the calculated linear dimensions of the strained region by more than about 20 per cent.

The essential conclusion of the present paper is, then, that the volume of the zone in which the material is near breaking-point in an extreme earthquake is at least of the order of the volume of a sphere of radius $25 \mathrm{~km}$. Allowing for uncertainties in the assumed values of $q, E, \mu$ and $S$, and taking into account the indications from other evidence, it is possible that the volume may reach that of a sphere of radius $50 \mathrm{~km}$. It is probably legitimate to apply (4) to the case of less extreme earthquakes, but it would appear that the value taken for $S$ should then be usually less than $10^{9} \mathrm{dyn} / \mathrm{cm}^{2}$

Finally, the writer wishes to thank Professors Gutenberg and Benioff for helpful discussion on various points in this paper, and for the use of the facilities of the Seismological Laboratory of the California Institute of Technology; and the United States Educational Foundation in Australia for kindly providing assistance in the form of a travel grant to California.

${ }^{6}$ H. Jeffreys, The Earth, 3rd ed. (Cambridge University Press, 1952), p. 196. 